\title{
Alterstice
}

Revue internationale de la recherche interculturelle

International Journal of Intercultural Research

Revista International de la Investigacion Intercultural

\section{Enjeux du vieillissement chez les Africains subsahariens en milieu francophone minoritaire canadien}

\section{Paulin Mulatris et Boniface Bahi}

Volume 4, numéro 1, 2014

URI : https://id.erudit.org/iderudit/1077482ar

DOI : https://doi.org/10.7202/1077482ar

Aller au sommaire du numéro

Éditeur(s)

Alterstice

ISSN

1923-919X (numérique)

Découvrir la revue

Citer cet article

Mulatris, P. \& Bahi, B. (2014). Enjeux du vieillissement chez les Africains subsahariens en milieu francophone minoritaire canadien. Alterstice, 4(1), 61-72. https://doi.org/10.7202/1077482ar
Résumé de l'article

Les enjeux du vieillissement des populations francophones d'origine africaine subsaharienne sont très peu connus. Une analyse de données qualitatives portant sur le vécu socioculturel et les déterminants de la santé, obtenues au moyen d'entrevues semi-structurées auprès d'immigrants francophones africains subsahariens des régions urbaines d'Edmonton et Calgary, permet de constater que cette population cumule des défis culturels et économiques qui les poussent à développer un mode de vie inapproprié aux conditions d'un vieillissement en santé. La nécessité que ces Néo-Canadiens développent des habitudes de vie adaptées mène à une réflexion sur les conditions socioéconomiques auxquelles ils font face et sur l'adaptation culturelle des soins. 


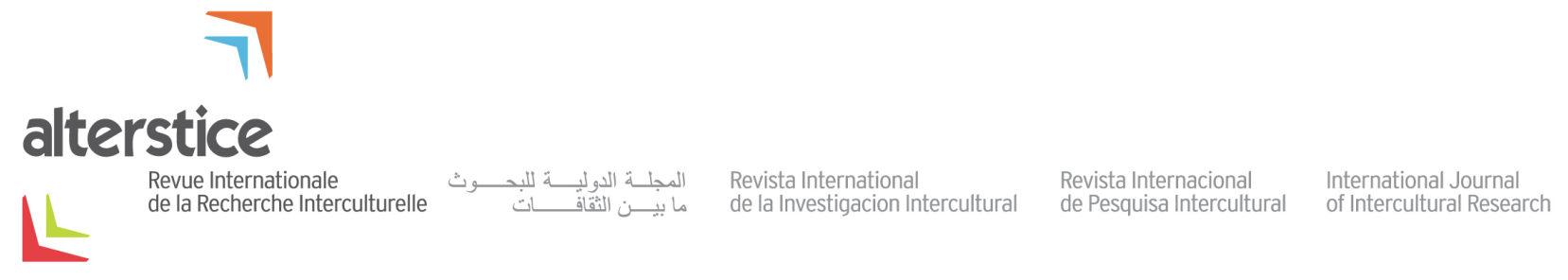

ARTICLE HORS THÈME

\section{Enjeux du vieillissement chez les Africains subsahariens en milieu francophone minoritaire canadien}

Paulin Mulatris ${ }^{1}$ et Boniface Bahi ${ }^{1}$

\section{Résumé}

Les enjeux du vieillissement des populations francophones d'origine africaine subsaharienne sont très peu connus. Une analyse de données qualitatives portant sur le vécu socioculturel et les déterminants de la santé, obtenues au moyen d'entrevues semi-structurées auprès d'immigrants francophones africains subsahariens des régions urbaines d'Edmonton et Calgary, permet de constater que cette population cumule des défis culturels et économiques qui les poussent à développer un mode de vie inapproprié aux conditions d'un vieillissement en santé. La nécessité que ces Néo-Canadiens développent des habitudes de vie adaptées mène à une réflexion sur les conditions socioéconomiques auxquelles ils font face et sur l'adaptation culturelle des soins.

\section{Rattachement des auteurs}

${ }^{1}$ Université de l'Alberta, Campus Saint-Jean, Edmonton (Alberta), Canada

\section{Correspondance}

paulin.mulatris@ualberta.ca

\section{Mots clés}

immigrants; francophones; Africains; vieillissement; santé

\section{Pour citer cet article :}

Mulatris, P. et Bahi, B. (2014). Enjeux du vieillissement chez les Africains subsahariens en milieu francophone minoritaire canadien. Alterstice, 4(1), 61-72. 


\section{Introduction}

La recherche qui fonde cette contribution s'articule autour de deux questions. Les conditions de vie et le mode de vie des immigrants francophones originaires d'Afrique subsaharienne ${ }^{1}$ installés au Canada, plus précisément en Alberta (Edmonton et Calgary), les préparent-ils à un vieillissement en santé2 ? Comment ces immigrants envisagent-ils leurs vieux jours? Parler de vieillissement pour une population dont la migration est parmi les plus récentes au Canada (Statistique Canada, 2011) peut sembler prématuré. En fait, mentionner que toute cohorte migratoire est l'affaire de jeunes adultes relève de l'évidence. Nous accordons donc plutôt un caractère prospectif et prédictif à notre démarche sur les perceptions de fin de vie des catégories sociales mentionnées. Notons que l'immigration en milieu francophone minoritaire du Canada est encore plus récente. Pourquoi vouloir documenter des enjeux de vieillissement et de santé auxquels sont confrontés les immigrants francophones africains adultes? Plusieurs raisons justifient ce choix. D'abord, vieillir en santé est un processus qui s'optimise toute la vie (Martel, Bélanger, Berthelot et Carrière, 2005). Pour demeurer en bonne santé durant ses vieux jours, il faut l'être en atteignant 65 ans. En ce sens, une connaissance du mode de vie des adultes permet d'anticiper les problèmes de santé des aînés (Thumerelle, 2000). De plus, ce travail, en tenant compte du phénomène de "l'effet de bonne santé des immigrants" (lié au constat que la santé des immigrants se détériorerait progressivement après leur arrivée au Canada, voir Ng, Wilkins, Gendron et Berthelot, 2005), permet d'intégrer les considérations immigrantes dans l'analyse des enjeux liés au vieillissement. Cette contribution permet alors non seulement de documenter et de faire avancer les connaissances sur le vieillissement des populations francophones africaines installées au Canada, mais aussi d'informer les praticiens et les politiques sur les enjeux sociaux globaux liés à l'immigration, au vieillissement et à la santé de ces populations.

\section{Cadre théorique}

L'augmentation de la longévité et la baisse du taux de fécondité font de la question du vieillissement un des défis auquel la plupart des sociétés occidentales font face. Comme la plupart des pays occidentaux, le Canada est concerné par ce phénomène, que Henripin (2005) présente comme l'un des principaux enjeux publics des prochaines années. De 1956 à 2005, l'espérance de vie à la naissance est passée de 75 à 82 ans pour la femme canadienne et de 68 à 78 ans pour l'homme. Sur cette même période, continuant sa descente sous le seuil de remplacement des générations ( 2,1 enfants par femme), l'indice de fécondité est passé de 3 enfants par femme à 1,51 (Statistique Canada, 2005). Ces changements enregistrés au niveau de l'espérance de vie et de l'indice de fécondité ont entraîné des modifications importantes au niveau de la structure par âge de la population et soustend, en quelque sorte, la politique d'immigration du Canada. Globalement, la proportion de personnes âgées (65 ans et plus) est à la hausse depuis 40 ans, au sens où elle est passée de $8 \%$ à $14 \%$ entre 1971 et 2011, et elle va se situer à $25 \%$ en 2036 selon Statistique Canada (2011). Selon les scénarios des projections faites par Statistique Canada (2005), entre $25 \%$ et $30 \%$ de la population canadienne sera composée de personnes âgées en 2056 . L'âge médian passerait de 39 à 45 ans.

Parmi les incidences du changement dans la structure de la population canadienne, les aspects sanitaires sont les plus considérés dans la littérature. Le vieillissement influence la demande de services de soins de santé, celle-ci se dégradant avec l'avancée en âge (Martel et collab., 2005; Morissette, Schellenberg et Silver, 2004; Pyper, 2006). Ce vieillissement met une pression sanitaire sur le système d'assurance-maladie universelle du Canada en termes de croissance continue des coûts. Le poids de l'âge se manifeste par des problèmes de solitude, de handicap incurable, de dépendance médicale et de prise en charge sociale (Thumerelle, 2000).

Comment les immigrants francophones africains se positionnent-ils par rapport à ces enjeux du vieillissement et à la santé, qui est vu comme un phénomène pluriel? De fait, la question du vieillissement de la population africaine est faiblement documentée. Les rares études disponibles sont sommaires et s'arrêtent sur les éléments statistiques

\footnotetext{
${ }^{1}$ Cette étude exploratoire a porté sur les populations africaines subsahariennes installées en Alberta. Dans la suite du texte, la désignation Africains se rapportera aux populations de cette origine.

${ }^{2}$ L'Organisation mondiale de la santé définit la santé comme " un état de complet de bien-être physique, mental et social, et [qui] ne consiste pas seulement en une absence de maladie ou d'infirmité ».
} 
sans analyser les défis spécifiques liés au vieillissement. On peut considérer les aînés africains vivant au Canada comme des " aînés en marge " s'inscrivant dans un processus de double minorisation lié à leur statut de minorité et conduisant à un vieillissement différentiel. Ils ne forment pas un effectif important et suscitent donc peu d'intérêt dans les études sur le vieillissement (Conseil consultatif sur le troisième âge, 2005). Ce n'est qu'au cours des deux dernières périodes intercensitaires que la population africaine a connu une forte croissance. Entre 1996 et 2001, cette population a augmenté de $32 \%$ pour représenter près de $1 \%$ du total de la population canadienne. Quoique faible, cette tendance à la croissance a aussi été observée en 2006 . Près de 10,6 \% des nouveaux arrivants au Canada étaient d'origine africaine, ce qui représentait une hausse de 8,3\% par rapport à 2001. Pour plusieurs raisons, dont principalement un accès facile aux services et la présence de réseaux familiaux, cette population s'installe dans les régions urbaines (Okonny-Myers, 2010). En Alberta, $24 \%$ (7 000) des nouveaux arrivants francophones installés dans la province depuis 1996 sont d'origine africaine (Association canadienne-française de l'Alberta [ACFA], 2011). Cette population africaine est relativement jeune par rapport à la structure par âge canadienne. En 2001, seulement $3 \%$ par les immigrants africains entraient dans la catégorie des aînés (65 ans et plus), alors que 58 \% faisaient partie de la population active ayant entre 25 et 65 ans (Lindsay, 2001).

En dépit d'une apparente globalité des enjeux de santé, les personnes âgées ne forment pas un groupe homogène. Plusieurs éléments (caractéristiques culturelles, situation financière, milieu de vie, histoire personnelle de vie, etc.) - rassemblés sous la notion de déterminants de la santé (Agence de la santé publique du Canada, 2011) particularisent ces enjeux publics du vieillissement et les réponses à y apporter. Dans le cas de la population africaine étudiée ici, la langue est un premier élément singularisant. Ces immigrants sont, comme tous les francophones vivant en contexte minoritaire, confrontés aux problèmes d'accessibilité aux soins de santé (Bowen, 2001; Ujimoto, 1995). Le rapport Romanow (2002) accorde à la notion d'accessibilité une acception très large : elle couvre des aspects relatifs non seulement au temps mais aussi aux facteurs sociaux et culturels qui interfèrent dans le rapport aux services et aux soins de santé. Dans le cas de l'Alberta, le système de santé étant construit selon le mode de vie de la majorité anglophone, le problème de l'accessibilité linguistique est un défi qui concernerait une proportion importante de francophones (Arabeyre, 2006; Lévesque, 2005 et 2006). Cependant, pour les immigrants francophones africains, au-delà de la question linguistique, des écueils de communication et de compréhension interculturelle demeurent. Au-delà, donc, des facteurs structurels, il y aurait des facteurs conjoncturels, disons, limitatifs pour les immigrants africains. En effet, dans un pays aussi culturellement diversifié que le Canada, les différences en matière de représentations culturelles (pensées, croyances, attitudes, valeurs) relatives à la santé, au système de santé et au vieillissement ne sont pas à sous-estimer. Elles peuvent constituer un obstacle dans le rapport soignés-soignants. Accéder aux services de soins de santé signifie, dans certains cas, accéder à une culture différente, celle du prestataire de ces soins (Desfossés, 1995; McPherson, 1995; Whalen, 1999). Mais aussi, en un sens inverse, le prestataire de soins de santé peut éprouver des difficultés à pénétrer l'univers culturel de son client. Cela conduit à une exigence de renouvellement de la formation des ressources humaines et de renouvellement également des ressources matérielles sanitaires du milieu médical accueillant (Bahi, 2007). Certains auteurs (Dilworth-Anderson et Gibson, 2002; Ujimoto, 1995) notent ce problème de représentations culturelles dans l'expression des difficultés psychologiques qui, selon eux, sont culturellement déterminées. Dans les cultures noires africaines, par exemple, les troubles de santé mentale sont un tabou. Cette attitude n'est pas de nature à favoriser leur traitement (Mbodie, 2001).

La pensée africaine par rapport à l'âge illustre l'importance des représentations culturelles dans l'approche des problèmes de santé et du vieillissement. Eboussi-Boulaga (1977) a relevé ainsi, dans les sociétés africaines, une organisation selon le sacré de l'origine. Cette organisation, qui ne pourrait être troublée sans perturber l'équilibre social, entraînerait un classement hiérarchique des êtres suivant leur proximité à l'origine divine, ancestrale. Ce rapport à l'origine s'inscrit au cœur du culte des ancêtres, à travers lequel tout aîné ayant eu une participation sociale enviable et déterminante pour la communauté devient un ancêtre (naissance dans l'au-delà) après sa disparition terrestre (Thomas, 1973). Dans certaines cultures, on s'attend à un retour (renaissance) terrestre de cet aîné-ancêtre à un moment ou à un autre de l'évolution de la communauté, à condition que la fin de vie ait lieu dans le contexte communautaire de l'origine. Cette forme de pensée conduit à valoriser les aînés qui occupent un rang hiérarchique important dans la famille et dans la société. Ils sont considérés comme les garants de la mémoire collective et de l'équilibre social (Bourdieu, 1963) car, comme la conscience populaire africaine le soutient, en Afrique, un vieillard qui meurt est une bibliothèque qui brûle. De fait, pour les aînés ayant un tel héritage culturel, 
la possibilité de vivre en résidence pour personnes âgées peut provoquer un sentiment d'exclusion et de disqualification sociales. La nature de l'estime de soi n'a-t-elle pas un impact sur la santé mentale individuelle? Ce type de positionnement social exige une prise en charge par la famille et n'est pas nécessairement transposable dans la société canadienne. Une étude menée parmi les immigrants haïtiens a démontré qu'une prise en charge familiale constitue un poids énorme pour les proches aidants immigrants (Ducharme, Paquet, Vissandjée, Carpentier, Lévesque et Trudeau, 2008). II n'est pas certain que la pensée qui soutient cette forme d'organisation sociale corresponde à l'ethos des sociétés occidentales modernes, qui valorisent la productivité économique et la performance au travail (Bourdieu, 1977). Dans ce cadre, c'est la contribution à la productivité économique du groupe qui définirait la position sociale des individus (Bourdieu, 1963 et 1977). La fin de la période active est donc susceptible de correspondre à une expérience de déclin et d'inutilité sociale. Le changement de contexte de vie et l'adaptation à la société canadienne peuvent mettre en péril l'idée d'une continuité culturelle sécurisante pour ces aînés africains, assurée, par exemple, par la validation de certaines sources africaines de prestige social. En fait, ces aînés seraient de véritables sources de mécanismes de transmission des savoirs aux plus jeunes générations (Bourdieu, 1963 et 1977). Or on note, comme effet du contexte socioculturel canadien au niveau linguistique, un fort taux d'anglicisation parmi les allophones (Castonguay, 2002). Ceux-ci auraient tendance à transmettre l'anglais comme langue maternelle à leurs enfants, et cette assimilation linguistique entraînerait donc des difficultés de communication entre les grands-parents ayant un héritage culturel africain à transmettre et leurs descendants anglicisés.

Le portrait économique de la population immigrante africaine est pour l'instant peu flatteur. Malgré des niveaux de scolarité élevés, plusieurs immigrants parmi nos enquêtés sont faiblement intégrés sur le plan socioprofessionnel, et ils réalisent peu de gains financiers (Gilmore, 2008; Gilmore et LePetit, 2008; Picot, Hou et Coulombe, 2007; Torczyner, 1997). Dans certains cas, l'âge tardif de l'immigration ou du premier emploi ne permet pas de constituer une épargne retraite conséquente. Cette situation de précarité financière exige une meilleure compréhension des mécanismes par lesquels un faible revenu influe sur les conditions de vieillissement en bonne santé de ces immigrants (Martel et collab., 2005). Dans le cas qui nous concerne, ces mécanismes doivent être examinés en tenant compte de leur intersectionalité avec les particularités culturelles de la population étudiée.

Le particularisme culturel et social des immigrants francophones africains participe, à l'évidence, à leur vieillissement différentiel. Cependant, ce vieillissement différentiel semble être maintenu et entretenu par certaines conditions sociales, psychologiques et économiques (Santerre et Letourneau, 1989). Dans l'ensemble, quel portrait peut-on dresser du vieillissement africain francophone au Canada, plus spécifiquement en Alberta?

\section{Méthodologie}

Les éléments présentés dans cet article proviennent d'une étude exploratoire qui visait à documenter et à déconstruire certaines perceptions sur les expériences de vie des immigrants francophones adultes d'origine africaine, installés dans les régions urbaines d'Edmonton et de Calgary, par rapport à leur vieillissement et à leur santé. Notre approche était qualitative, interprétative, narrative et comparative (Cipriani, 2010). Comme l'étude envisagée engageait les chercheurs à entrer en contact avec des sujets humains, les autorisations éthiques nécessaires ont été obtenues en conformité avec les normes de l'Université de l'Alberta. Nous nous sommes appuyés sur les réseaux d'associations communautaires africaines locales pour présélectionner les participants en fonction de quelques critères, dont l'âge, le genre, le niveau de scolarité, le statut professionnel, l'expérience du système de soins de santé, le réseau familial et le pays d'origine. Les listes des participants disponibles fournies par ces organismes communautaires nous ont permis de retenir 25 participants immigrants francophones (parmi lesquels 3 femmes) et 2 intervenants en santé. Ce groupe a été constitué de sorte à inclure différentes catégories d'âge parmi les personnes susceptibles d'être concernées par l'objet de la recherche. Tous les participants avaient un niveau d'études post-secondaires.

Deux techniques qualitatives de collecte des données ont été utilisées. En mars 2006, deux groupes de 12 enquêtés, répartis chacun en deux groups de discussion (focus group) de 6 participants chacun, ont été organisés à Edmonton et à Calgary. Les éléments recueillis pendant ces groupes de discussion renvoyaient essentiellement aux déterminants de la santé présentés par le réseau canadien de santé et commentés dans 
l'étude de Martel et collab. (2005). Mais, pour tenir compte du caractère englobant du phénomène étudié (Munchielli, 1991), nous avons rajouté à ces déterminants des éléments psychosociaux qui spécifiaient les thèmes en discussion par rapport au contexte de vie d'une population immigrante. Nous avons ainsi adapté et intégré dans le guide d'entretien des éléments portant sur les facteurs socioéconomiques (niveau de scolarité, revenu), les habitudes de vie à l'égard de la santé (tabagisme, activité physique), les facteurs psychosociaux (stress, soutien social), les problèmes de santé chroniques et les consultations médicales.

Aux mois d'avril et de mai 2007, 25 récits de vie ont été recueillis au moyen d'entrevues semi-dirigées d’une durée de 90 minutes avec des immigrants francophones des régions d'Edmonton et de Calgary (tableau 1). Ces entrevues visaient à recueillir, auprès des participants, des perspectives un peu plus approfondies sur le vieillissement, alors que les entrevues avec les deux prestataires des soins de santé visaient davantage les enjeux de santé touchant leur clientèle immigrante africaine. La méthode des récits de vie nous a permis de développer une approche plus attentive à la singularité des vécus des participants.

Tableau 1. Caractéristiques des participants africains à l'étude

\begin{tabular}{lc}
\multicolumn{1}{c}{ Caractéristiques } & Effectifs ( $\mathbf{~ = ~ 2 3 ) ~}$ \\
\hline Genre & 3 \\
Femmes & 20 \\
Hommes & \\
Âge & 4 \\
Force de l'âge (25 à 44ans) & 17 \\
Âge mûr (45 à 64 ans) & 2 \\
Aînés (65 ans et plus) & \\
Pays d'origine & 8 \\
Congo (RDC) & 4 \\
Rwanda & 3 \\
Burundi & 4 \\
Cameroun & 2 \\
Sénégal & 2 \\
Côte d'lvoire & \\
\hline
\end{tabular}

Le canevas d'entrevue n'a pas été suivi à la lettre et le critère de proximité culturelle avec les personnes interrogées a été utilisé dans le choix de l'enquêteur, lui aussi d'Afrique subsaharienne (Tremblay, 1991). Ce choix a permis aux participants de puiser dans leurs expériences de vie pour faire ressortir leurs perspectives par rapport aux thèmes en discussion. Les données de ces entrevues ont été transcrites et décortiquées manuellement suivant une analyse de type phénoménologique et comparative, ce qui nous a permis de faire émerger la perspective des participants par rapport au vieillissement, à leur mode de vie et àleur santé (Boudon, 2012; Quivy et Campenhoudt, 2006; Seron, 2001). Pour préserver la confidentialité des données liées aux participants, nous avons utilisé des initiales pour les identifier dans la transcription des récits de vie.

\section{Le discours des immigrants africains sur leur santé et leurs vieux jours}

Plusieurs thèmes ont émergé des discours des participants. Ceux-ci ont été rassemblés en fonction des éléments relevant des principaux déterminants de la santé : la culture (langue, alimentation), l'activité physique, le revenu, les habitudes de vie, les visites médicales et le réseau familial. Pour la plupart de ces thèmes, ce sont surtout des problèmes d'intégration ou d'exclusion susceptibles d'affecter la santé des répondants qui ont été mentionnés.

\section{Les difficultés d'intégration}

L'intégration sociale dans la société canadienne est un défi vécu diversement par les immigrants. Si pour certains l'arrimage à la société d'accueil s'est relativement bien effectué, pour d'autres les difficultés rencontrées paraissent 
insurmontables. Les problèmes linguistiques, de reconnaissance des diplômes et compétences et les différences culturelles sont une source d'inconfort psychologique.

L'intégration, c'est un des grands défis que j'ai affrontés ici au Canada. D'abord il a fallu que je m'adapte à tout de la culture canadienne, que je cherche du travail, que je cherche une maison, que je m'adapte au climat. J'ai dû faire beaucoup de volontariat, j'ai dû faire des travaux dans des «factories » et donc quelque chose que je n'avais pas fait dans ma vie. Quand je suis venu ici en Alberta j'ai travaillé avec PDD [Pervasive Developmental Disorder] donc des personnes avec problèmes somatiques et puis j'ai dû étudier aussi. (C. K., 48 ans)

Satisfaire le quotidien familial force ces immigrants à évoluer dans des contextes professionnels qui, de manière générale, ne correspondent pas à leur formation et à leur expérience professionnelle antérieure. L'exigence d'augmenter le revenu familial impose et justifie des journées ressenties comme longues par certains répondants. Le manque de temps est un sentiment généralement partagé par les personnes interrogées. Ce rythme de vie affecte le temps de sommeil et engendre du stress, un facteur psychosocial récurrent :

De lundi à vendredi je pars au travail à 8 heures, et puis je redescends à 8 heures, parfois à 9 heures. Chaque fois, j'ai à peu près 11 heures ou 12 heures de travail. Je reviens à la maison fatiguée, je mange et je dors. (A. B. A., 26 ans)

Ma vie professionnelle connaît beaucoup de problèmes ces derniers temps. J'avais un emploi que j'ai quitté après 3 mois. Je l'ai quitté pour occuper un autre emploi qui donnait plus d'argent, et plus de défis mais que j'ai perdu au bout de 2 semaines. Ça, ça m’a donné énormément de stress, jusqu'à maintenant d'ailleurs. Actuellement je suis sur appel dans l'enseignement. (V. M., 53 ans)

Il n'est pas toujours facile de concilier sa culture d'origine avec celle du milieu d'accueil, au sens de choc culturel. Des répondants disent préférer préserver quelques aspects culturels d'origine tout en s'efforçant d'adopter certaines valeurs de la culture canadienne, une réponse à leur choc culturel, ce qui se traduit sur le plan de I'alimentation. Si dans l'ensemble les répondants disent avoir une alimentation plus ou moins saine, celle-ci est principalement africaine. Les répondants affirment ne pas parvenir à s'adapter à la nourriture canadienne malgré le temps écoulé depuis leur arrivée.

Malgré mes 12 ans au Canada, la seule chose pour laquelle je n'ai pas encore immigré, c'est au niveau de la nourriture. J'aime manger la nourriture de chez moi : les feuilles de manioc, je mange le poisson. La nourriture que j'aime manger prend beaucoup de temps à cuire et sans cette nourriture je mange mal. (B. N., 35 ans)

\section{Généralement en santé}

Les entrevues révèlent un niveau de scolarité postsecondaire chez les répondants. Ils disent être en bonne santé et avoir une bonne connaissance du système médical canadien et albertain. Les médias (télévision, journaux, Internet) leur sont utiles pour développer leur culture dans le domaine médical. Le docteur D., intervenant communautaire interrogé dans le cadre de cette enquête, relativise cette connaissance du système de santé canadien par ces immigrants. Selon lui, une mauvaise connaissance subsiste et contribuerait, par exemple, à la difficulté à faire des suivis médicaux.

Autre chose c'est de naviguer dans un système de santé qu'on ne comprend pas, qu'on connaît moins bien. Parce que, quand on arrive dans un endroit où le système de santé fonctionne d'une autre manière, [cela signifie] de pas comprendre c'est où, c'est quoi le coin d'accès privilégié, et c'est quoi la manière qu'on peut faire les choses, c'est quoi le procédé, comment est-ce qu'on obtient un rendez-vous, pourquoi ne pas manquer de rendez-vous, où est ce qu'on va chercher nos vaccins, toutes ces choses là. (Docteur D.)

De fait, il nous est apparu que, même si tous nos répondants ont un médecin traitant, le suivi médical ne semble pas être une pratique commune: on va voir le médecin quand c'est grave (Dedy et Saky, 1994). Pourtant, des problèmes d'obésité et de pathologies chroniques sont présents :

Je ne peux pas dire que je suis en bonne santé puisque je suis obèse. II m'arrive de changer le régime de temps en temps. Par exemple, j'ai adopté un autre régime encore très rigoureux et j'aimerais éliminer tout ce qui est gras, glucide même en quantité. Ça fait une semaine que j'applique ce nouveau régime, je ne me sens pas mieux qu'avant. (L. L., 47 ans) 
[...] au niveau de la santé physique comme moi personnellement je suis à risque parce que je suis diabétique de type II. (O. R., 48 ans).

Les propos de l'un des responsables du réseau francophone albertain de santé confirment les impacts sanitaires des difficultés socioéconomiques rencontrées par ces immigrants :

Il y a des difficultés au niveau de l'intégration socioéconomique : il y a plusieurs diplômés ayant appris une discipline, qui viennent de l'Afrique, qui ont de la difficulté à faire reconnaître leurs diplômes, et donc ça limite leur emploi, et leurs revenus. Ce qui a un impact à moyen terme sur leur statut social, le statut économique, et évidemment ces facteurs là ont aussi des impacts sur la santé. (L. T., responsable)

Ces difficultés sont aussi liées aux habitudes de vie des répondants. Pourtant, de manière générale, l'alcool et la cigarette ne font pas partie de leurs habitudes de vie. Dans bien des cas, c'est la religion qui est évoquée comme raison principale poussant à s'abstenir de fumer ou de consommer de l'alcool (Bibeau, 2002).

Je suis ce qu'on appelle religieux, protestant évangéliste. Donc la cigarette, l'alcool, vraiment, je ne suis pas là. Je prends un peu de vin quand même, si je mange, je peux prendre du vin. L'alcool, la drogue, la cigarette là, moi je suis absent. (W. M., 31 ans)

Malgré l'intérêt manifeste pour les activités physiques, les difficultés financières et le manque de temps favorisent l'inactivité physique selon les répondants. Occasionnellement, certains pratiquent la marche, d'autres font du vélo. Ce problème d'inactivité semble être plus prononcé pour les femmes que pour les hommes.

J'aime beaucoup l'activité physique : je fais du vélo pendant cette période-ci. Normalement, j'avais une activité physique régulière, j'avais une carte du YMCA, je faisais du squash, je faisais du work-out. Je n'ai pas pu renouveler mon abonnement au YMCA. J'ai eu une période d'inactivité de presque 10 années, parce que je ne pouvais pas renouveler ma carte et je n'avais pas assez d'argent pour payer un abonnement régulier. C'est tout à fait cher. (V. M., 53 ans)

\section{L'Église et la famille, des soutiens importants}

La famille et l'Église occupent une place prépondérante dans la vie de ces immigrants et, en l'absence de membres de leur famille dans la ville où ils habitent, ces immigrants s'appuient sur l'Église, qui leur offre un soutien social important. La religion donne sens à leur vie et leur permet de trouver une paix intérieure.

Je suis marié, et j'ai des enfants que j'ai adoptés. Ma femme a des enfants. J'ai un enfant biologique. Je suis plutôt genre famille. J'ai toujours vécu avec les enfants. Je sors d'une famille nombreuse, ce qui fait que je suis toujours entouré de pas mal de monde. Alors, c'est ça la vie familiale. (V. M., 53 ans)

Quand on se réveille le matin, on s'apprête comme tout le monde. On commence avec quelques minutes de prière. Moi je prie dans ma douche parce que je n'ai pas assez de temps pour prier, et après j'apprête les enfants et quand ils prennent l'autobus moi je vais au travail où je fournis le meilleur de moi-même. Je sais que c'est ma croix; si Dieu me l'a donnée, c'est que je suis capable. (B. N., 35 ans)

\section{Vieillir en Afrique ou au Canada?}

Le doute est palpable quand on demande aux participants s'ils ont un choix sur leur lieu de vie à la retraite. Dans l'ensemble, ceux qui ne pensent pas retourner au pays d'origine préfèrent être pris en charge par leurs enfants au Canada. Plusieurs raisons sont évoquées pour justifier ce choix : les difficultés financières, le climat, l'alimentation, le refus d'habiter un foyer pour personnes âgées et la volonté de rester en famille, l'inadaptation des soins dans les centres pour personnes âgées et la perception que le soutien des enfants soit une police d'assurance pour les vieux jours. Retourner en Afrique pourrait être vu comme un échec de la migration par certains proches en Afrique, bien qu'une tendance philopatrique - attachement au territoire d'origine, plus marquée pour les femmes selon Chapais (2008) - soit constatée chez les immigrants. Aussi, dans un contexte de conjonctures socioéconomiques et sanitaires peu favorables dans les pays d'origine, il est pertinent de se demander si cette philopatrie tient encore. 
De fait, l'insuffisance des revenus amassés constitue un obstacle majeur au développement d'une capacité d'autofinancement pour la retraite et les vieux jours. Conscients de cette difficulté, quelques répondants évacuent la question de la retraite comme n'étant pas au chapitre de leurs préoccupations actuelles. D'autres évitent le sujet et ne veulent pas y penser.

Ça c'est quelque chose qui me tracasse énormément parce que depuis plusieurs années, je ne me vois pas vivre au Canada pour mes vieux jours : je ne voudrais pas être dans les maisons de retraite pour personnes âgées. Je voudrais rentrer chez moi parce que justement n'ayant pas d'épargne retraite et donc je ne sais pas comment je vais pouvoir vivre, vivre dans la pauvreté je préfère peut-être rentrer chez moi plutôt que de rester ici parce que je n'ai pas cotisé pour avoir une bonne somme pour m'assurer au niveau de la retraite. (O. R., 48 ans)

Les vieux jours, au Canada, ça c'est une question à voir. Des fois, il faut commencer à y penser, parce que le pays qu'on choisit là, on finira nos vieux jours ici là. Si c'est finir nos jours dans des foyers pour personnes âgées, alors là à ce moment on se pose toujours la question de savoir comment on sera traité là bas, ça c'est quelque chose qu'on est en train de voir. (J. L., 48 ans)

Pour le docteur D., la prise en charge des aînés par les enfants n'est pas toujours un choix approprié. Des problèmes de communication et d'interprétation compromettent parfois l'offre de soins.

En vieillissant, on devient de plus en plus vulnérable et moins autonome, plus dépendant des autres. On est vraiment désavantagé quand on négocie nos soins dans une langue qui n'est pas la nôtre. Et c'est toujours difficile aussi d'essayer de faire ces choses là à travers nos enfants. Parce que nos enfants, des fois ce n'est pas le meilleur filtre de ces informations là. Et alors, ce qui arrive souvent à toutes les communautés minoritaires où les aînés ne parlent pas anglais ou parlent moins bien anglais, et puis les enfants parlent bien, c'est que toutes les histoires ne sont pas bien connues, ne sont pas bien expliquées, et ça devient difficile. (Docteur D.)

Une proche aidante qui vit avec une aînée en situation de dépendance commente les difficultés :

Je vis avec une belle-maman qui est paralysée et qui a perdu son langage et je dois vous avouer que parfois c'est dur; il y a des rendez-vous dans les hôpitaux, des traductions à faire pour elle parce qu'elle ne parle plus. II y a tout le stress que l'on vit avec une personne malade. Même si on dit que c'est une paralysie, il y a des choses qu'ils ne comprennent pas. (B. N., 35 ans)

\section{Discussion}

Il se dégage de l'analyse des propos des répondants, au-delà des difficultés partagées par tous les francophones en situation minoritaire (accessibilité linguistique aux soins de santé), quelques facteurs particuliers qui pourraient avoir un impact sur le vieillissement en bonne santé des immigrants francophones africains. Parmi ceux-ci, les aspects socioéconomiques et culturels (pensées sur le vieillissement, habitudes alimentaires, tabous sur la santé mentale, etc.) semblent jouer un rôle déterminant. La difficulté à se trouver un emploi adapté à leur niveau de scolarité et procurant un revenu décent affecte leur statut socioéconomique et du même coup leur santé mentale et physique. Dans les faits, il y a un véritable écart entre les aspirations pré-migratoires et le niveau concret de réalisation de celles-ci (Bahi, 2007). L'immigrant ne se serait pas ajusté à ses projets au sens où ce qu'offre la réalité de vie en milieu immigrant semble loin de ses attentes. Cette dissonance cognitive reposant sur l'inadéquation entre les attentes et ce que la réalité du contexte immigrant impose constituerait la plus grande source de stress. En effet, si seulement quelques individus ont signalé des problèmes d'hypertension ou de diabète, la majorité de nos répondants n'ont pas caché leur état de stress permanent essentiellement causé par la situation de précarité matérielle dans laquelle ils évoluent. Ces éléments ont tendance à confirmer "l'effet de la bonne santé des immigrants » révélé par Ng et collab. (2005), car les plus anciens d'entre eux semblent être ceux qui expérimentent le plus de problèmes liés au stress, à l'hypertension ou à l'obésité. Ainsi, même s'ils semblent avoir de bonnes habitudes de vie vis-à-vis du tabagisme ou de la consommation d'alcool, très peu pratiquent une activité physique. À cela s'ajoutent des problèmes de négligence en matière de suivi médical. Pourtant, celui-ci est jugé indispensable pour les adultes d'âge mûr et conditionne, dans une certaine mesure, un vieillissement en bonne santé (Martel et collab., 2005). 
En général, le niveau de scolarité est un déterminant de la santé important parce qu'il ouvre, a priori, sur la réussite sociale et sur de meilleures conditions de vie (Pérusse, 1988). Cependant, cette corrélation entre niveau d'études élevé et réussite sociale ne se vérifie pas pour les participants à notre étude. Les facteurs explicatifs sont de plusieurs ordres, mais le principal est la non-reconnaissance des expertises et le refus qu'elles soient mises à profit dans le système de travail canadien (Belkhodja et collab., 2009; Mulatris, 2010). Cette situation semble priver certains immigrants africains francophones de support à leur qualité de vie. En effet, d'une part, quoique bien informés sur l'impact de divers facteurs (alimentation, activité physique, habitudes de vie, etc.) sur leur santé physique, la plupart de ces immigrants vivent dans un environnement social qui ne favorise pas ces comportements ou les choix appropriés au maintien d'une bonne santé. D'autre part, certaines stratégies (faible suivi médical, manque de pratique sportive, etc.) mises de l'avant par ces immigrants semblent inadaptées à l'objectif d'un vieillissement en santé. On peut se demander ce que seraient les impacts de leur vie en milieu rural francophone, réputé moins doté en équipement sociosanitaire que les milieux anglophones.

Par ailleurs, l'insistance des répondants à parler des éléments linguistiques traduit plus généralement un appel à l'accessibilité culturelle aux soins de santé. En ce sens, la prestation des soins de santé est une rencontre culturelle.

Les institutions doivent comprendre que les soins doivent s'adapter : il faut embaucher et mettre en place des personnes qui sont mieux aptes à pouvoir intervenir convenablement. Elles doivent arrêter d'insister sur le fait qu'on embauche les meilleurs gens avec les meilleures aptitudes à travailler sans tenir compte du contexte culturel dans lequel ces gens-là travaillent. Le meilleur candidat devrait être la personne qui est capable de faire culturellement interférence avec les clients. (Docteur D.)

En effet, les aspects culturels (spiritualité, famille, nourriture) occupent une place importante dans la vie de ces immigrants. Ils leur fournissent une base leur permettant de retrouver, en dépit d'un contexte socioéconomique instable, une cohérence dans leur vie. En ce sens, plusieurs de nos répondants perçoivent la prise en charge familiale ou le retour vers le pays d'origine comme une solution permettant de contourner les défis culturels et financiers qui les guettent au troisième âge. Cependant, les analyses de Castonguay (2002) et de Ducharme et collab. (2008) révèlent que les familles expérimentent aussi des difficultés de continuité culturelle et de prise en charge qui les empêcheraient de fournir un support complet aux membres qui en ont besoin. Dans ce cas, et en référence à Waldorf (1995), si on retient le caractère dichotomique d'une vie immigrante marquée par la recherche constante d'un équilibre entre l'attachement au pays d'origine et l'intégration au pays de destination, on peut penser que les difficultés d'intégration pousseraient certains immigrants à relativiser la perspective d'un vieillissement au Canada. Ce choix du lieu de retraite ne change pourtant rien à la réalité d'une vie active marquée par la précarité et qui ne présage pas un vieillissement en santé.

Nous ne saurions manquer de relever quelques limites à ce travail. Son caractère exploratoire relativise la portée des analyses, bien que ces dernières touchent des paramètres importants du vieillissement des populations. À ce constat s'ajoute le fait que des ressources financières limitées ont justifié le choix de l'espace albertain comme seul champ d'étude pour la sélection des participants à la recherche, alors que notre perspective embrassant les conditions quotidiennes de vie suggère de varier les provinces d'étude pour comparer l'offre en services sociosanitaires et des en ressources d'aide. Enfin, les pays d'origine des participants auraient pu être plus variés. Ce sont là quelques limites qu'une recherche future pourrait réussir à combler.

\section{Conclusion}

Cette étude est exploratoire. Son but était à la fois de mieux comprendre les impacts du mode de vie des immigrants francophones africains sur leur vieillissement en santé et le choix de leur lieu de vie après leur période de vie active. Une revue de la littérature a permis de situer cette question dans le contexte du vieillissement des populations francophones au Canada. Dans ce cadre, les défis de santé et les problèmes linguistiques figurent au premier plan des difficultés rencontrées. L'examen des déterminants de la santé en rapport avec le vécu socioculturel des immigrants francophones africains a permis de constater qu'ils cumulent des défis culturels et économiques qui auraient tendance à hypothéquer un vieillissement en santé. Ces défis culturels et économiques sont à relier au phénomène de "l'effet de bonne santé des immigrants » identifié par Ng et collab. (2005). La sensibilisation à la nécessité de développer des habitudes de vie adaptées à une vie et à un vieillissement en santé 
pourrait prendre la forme d'une réflexion sur les conditions socioéconomiques de ces immigrants et sur l'adaptation culturelle des soins. Plusieurs participants ont exprimé leur choix de demeurer dans un cadre familial après leur vie active. Cependant, les implications d'un tel choix n'ont pas été totalement examinées dans cette étude. D'autres recherches sont nécessaires, dans le cadre d'un examen général de la situation des aînés au Canada, pour mieux comprendre les impacts de ces choix dans un contexte culturel différent de celui de la société d'origine de ces immigrants.

\section{Références bibliographiques}

Agence de la santé publique du Canada (2011). Qu'est-ce qui détermine la santé? [en ligne]. http://www.phacaspc.gc.ca/ph-sp/determinants/index-fra.php

Arabeyre, C. (2006). Préparer le terrain pour la santé en français. Edmonton : Réseau santé albertain.

Association canadienne-française de l'Alberta (ACFA) (1991). Stratégie 2030 : un plan d'action communautaire. L'immigration francophone en Alberta. Edmonton : ACFA.

Bahi, B. (2007). Dérives et réussite sociale en Afrique. Paris : L'Harmattan.

Belkhodja, C., Forgues, É., Gaboury, I., Guignard, J., N., Bahi, B., Nkolo, C., et Tawil, N. (2009). L'intégration des diplômés internationaux en santé francophones dans les communautés francophones en situation minoritaire [en ligne). Institut canadien de recherche sur les minorités linguistique et Consortium national de formation en santé. http://cnfs.net/fr/download.php?id=716

Bibeau, G. (2002). Dieux étrangers, société post-religieuse et clinique. Prisme, 38, 60-82.

Boudon, R. (2012). Aux racines de la " bonne sociologie ». Revue internationale de systémique complexe et d'études relationnelles. Nouvelles perspectives en sciences sociales, 8(1), 119-161.

Bourdieu, P. (1977). Algérie 60. Structures économiques et structures temporelles. Paris : Minuit.

Bourdieu, P. (1963). La société traditionnelle; attitude à l'égard du temps et conduite économique. Paris : Sociologie du travail.

Bowen, S. (2001). Barrières linguistiques dans l'accès aux soins de santé. Ottawa : Santé Canada.

Castonguay, C. (2002). Assimilation linguistique et remplacement des générations francophones et anglophones au Québec et au Canada. Recherches sociographiques, 43(1), 149-182.

Chapais, B. (2008). Primeval Kinship. Cambridge, MA : Harvard University Press.

Cipriani, R (2010). L'analyse qualitative comme approche multiple. Dans J. Hamel (dir.) L'analyse qualitative interdisciplinaire. Définitions et reflexions (p. 29-45). Paris : L'Harmattan.

Conseil consultatif national sur le troisième âge (2005). Aînés en marge : Les aînés des minorités ethnoculturelles [en ligne]. Ottawa : Conseil consultatif national sur le troisième âge. publications.gc.ca/collections/Collection/H88-5-1-2005F.pdf

Dedy, S. et Saky, Z. (1994). Rites funéraires et santé publique en Afrique (rapport de recherche). Abidjan : Université d'Abidjan.

Desfossés, D. (1995). Gérer la diversité dans le réseau socio-sanitaire du Québec. Info ressources humaines, 17(14), 8-9.

Dilworth-Anderson, P. et Gibson, B (2002). The cultural influence of values, norms, meanings and perceptions in understanding dementia in ethnic minorities. Alzheimer's disease and associated disorders, 16(52), 856-863.

Ducharme, F, Paquet, M, Vissandjée, B., Carpentier, N., Lévesque, L. et Trudeau, D. (2008). Des services à domicile culturellement sensibles : perspective des intervenants et des proches-aidantes originaires d'Haïti en tant que cas traceur. Canadian Journal on Aging / La Revue canadienne du vieillissement, 27(2), 191-205.

Eboussi-Boulaga, F. (1977). La crise du Muntu. Authenticité africaine et philosophie. Paris : Présence africaine. 
Gilmore, J. (2008). Les immigrants sur le marché canadien du travail en 2006 : analyse selon la région ou le pays de naissance [en ligne]. Ottawa : Statistique Canada. ( $\left.\mathrm{n}^{\circ} 71-606-\mathrm{X} 2008002\right)$

Gilmore, J. et LePetit, C. (2008). Les immigrants sur le marché du travail canadien en 2007 : analyse selon la région d'obtention des études postsecondaires. Ottawa : Statistique Canada. ( $\left.\mathrm{n}^{\circ} 71-606-\mathrm{X}\right)$

Henripin, J. (2005). Dénatalité, vieillissement et immigration : Les défis d’une démographie changeante. Options politiques, mars-avril, 62-66.

Lévesque, A. (2005). La santé des aînés francophones en milieu minoritaire : services, défis et obstacles. Winnipeg : Collège universitaire de Saint-Boniface.

Lévesque, A. (2006). Langue et santé : La situation des francophones en milieu minoritaire. Winnipeg : Collège universitaire de Saint-Boniface

Lindsay, C. (2001). Profils de communautés ethniques au Canada : La communauté africaine au Canada. Ottawa : Statistique Canada. ( $\left.n^{\circ} 89-621-\mathrm{XIF}, 10\right)$

Martel, L., Bélanger, A., Berthelot, J.-M. et Carrière, Y. (2005). Vieillir en santé. En santé aujourd'hui, en santé demain? Résultats de l'enquête nationale sur la santé de la population. Ottawa : Statistique Canada. $\left(\mathrm{n}^{\circ} 82-\right.$ 618-MWF2005004)

Mbodie, N. (2001). Pour une politique de santé mentale adaptée en Afrique noire. Médecine d'Afrique noire, 48(11), 465-471.

McPherson, B. (1995). Aging from a historical and comparative perspective: Cultural and subcultural diversity. Dans R. Neugebauer-Visano (dir.), Aging and inequality: cultural constructions of differences (p. 31-77). Toronto : Canadian Scholars'Press.

Morissette, R., Schellenberg, G. et Silver, C. (2004). Inciter les travailleurs âgés à rester au poste, L'emploi et le revenu en perspective, 5(10), 16-22.

Mulatris, P. (2010). Disqualification professionnelle et expériences temporelles : enquête auprès des immigrants francophones africains installés en Alberta, British Journal of Canadian Studies, 23(1), 73-86.

Munchielli, A. (1991). Les méthodes qualitatives. Paris : Presses universitaires de France.

Ng, E., Wilkins, R., Gendron, F. et Berthelot, J.-M. (2005). L'évolution de l'état de santé des immigrants au Canada: constats tirés de l’Enquête nationale sur la santé de la population. Ottawa : Statistique Canada. (n 82-6148MWF2005002)

Okonny-Myers, I. (2010). Mobilité interprovinciale des immigrants au Canada. Ottawa : Citoyenneté et Immigration Canada.

Organisation mondiale de la santé (OMS) (1946). Préambule. Dans Constitution de l’Organisation mondiale de la santé. New York : OMS.

Pérusse, D. (1988). Succès social et succès reproductif dans les sociétés modernes : Une analyse sociobiologique. Anthropologie et sociétés, 12(3), 151-174.

Picot, G, Hou, F. et Coulombe, S. (2007). Le faible revenu chronique et la dynamique du faible revenu. Ottawa : Statistiques Canada. ( $n^{\circ} 11 \mathrm{F0019MIF)}$

Pyper, W. (2006). Vieillissement, santé et travail. L'emploi et le revenu en perspective, 7(2), 5-17.

Quivy, R. et Campenhoudt, L.-V. (2006). Manuel de recherche en sciences sociales. Paris : Dunod.

Romanow, R. (2002). Guidé par nos valeurs : l'avenir des soins de santé au Canada-Rapport final. Saskatoon : Commission sur l'avenir des soins de santé au Canada.

Santerre, R., et Létourneau, G. (dir.) (1989). Vieillir à travers le monde, contribution à une gérontologie comparée. Québec: Presses de l'Université Laval. 
Seron, D. (2001). Introduction à la méthode phénoménologique. Bruxelles : De Boeck Université.

Statistique Canada (2005). Recensement du Canada : projections démographiques pour le Canada, les provinces et les territoires 2005-2031. Ottawa : Statistique Canada. ( ${ }^{\circ}$ 91-520-X)

Statistique Canada (2011). Enquête nationale auprès des ménages. Ottawa : Statistiques Canada ( ${ }^{\circ}$ 99-010X2011001)

Thomas, L.-V. (1982). La mort africaine. Idéologie funéraire en Afrique Noire. Paris : Payot.

Thomas, L.-V. (1983). Le pluralisme cohérent de la notion de personne en Afrique noire traditionnelle. Dans G. Dieterlen (dir.), La notion de personne en Afrique noire (p. 340-420). Paris : Centre national de la recherche scientifique.

Thumerelle, J.-P. (2000). Longévité, vieillissement et santé [en ligne]. Dans les Actes du FIG 2000 à http://archivesfig-st-die.cndp.fr/actes/actes_2000/thumerelle/article.htm

Torczyner, J. (1997). Diversity, mobility and change: the dynamics of Black communities in Canada. Montréal : McGill Consortium for Ethnicity and Strategic Social Planning

Tremblay, A. (1991). Sondages : histoire, pratique et analyse. Boucherville : Gaétan Morin.

Ujimoto, V. (1995). The ethnic dimension of aging in Canada. Dans R. Neugebauer-Visano (dir.), Aging and inequality: cultural constructions of differences (p. 3-29). Toronto : Canadian Scholars' Press.

Waldorf, B. (1995). Determinants of international return migration intentions. Professional geographer, 47(2), 125136.

Whalen, D. (1999). Cultural sensitivity. A matter of respect. Canadian Nurse, 95(9), 43-44. 